\title{
Pain Perception after Periodontal Therapies
}

\author{
Dr. Shaili Pradhan, ${ }^{1}$ Dr. Rejina Shrestha, ${ }^{1}$ Dr. Ranjita Shrestha Gorkhali ${ }^{1}$ \\ 'Periodontology and Oral Implantology Unit, Department of Dental Surgery, \\ National Academy of Medical Sciences, Bir Hospital, Kathmandu, Nepal
}

\begin{abstract}
Background: Pain perception is a complex sensory experience which is perceived by different individuals in different manners. The pain perceived by the patient after periodontal surgery may vary based on different parameters.

Aim: This observational study was conducted to evaluate the perception of pain after periodontal therapies.

Materials and Methods: A total of 63 surgeries were carried out in 50 patients and the surgeries were divided into three categories: open flap debridement, resective and regenerative surgeries, and periodontal plastic surgeries. The pain experienced by the patient was recorded on the visual analog scale that ranged from 0 to 10 .

Results: The mean VAS score for different periodontal surgeries was 2.49. The study showed highest mean VAS in open flap debridement (2.74) followed by periodontal plastic surgery (2.5) and the lowest in resective and regenerative procedures (2.13). Among various variables such as age, sex, periodontal dressing, arch, amount of local anaesthesia and time duration, the data showed statistical difference between VAS score and sex $(\mathrm{p}=0.04)$ and between VAS score and amount of local anaesthesia $(\mathrm{p}=0.012)$.

Conclusion: The study showed there is low pain perception after different periodontal surgeries as measured by VAS. Proper understanding of the variables that affect pain is important as they may produce emotional responses that could influence compliance and the therapy result. Inadvertent use of large dose of anaesthetics beforehand assuming high anticipation of pain should be discouraged as the increase in volume relates to increased pain. .

Keywords: Anxiety; pain perception; periodontal therapy; visual analog scale.
\end{abstract}

\section{INTRODUCTION}

The International Association for the Study of Pain (IASP) has defined pain as an unpleasant sensory or emotional experience associated with actual or potential tissue damage or described in terms of such damage. ${ }^{1}$

Periodontal diseases are mostly chronic inflammatory conditions that cause destruction of periodontium. Treatment usually ranges from simple scaling and root planing (SRP/ non-surgical treatment) to more advanced surgical procedures. ${ }^{2}$ Numerous questions arise when patients are about to undergo dental treatment, main concern is the fear of pain. As pain influence informed consent and patient management, dental practitioner performing treatment is also concerned. ${ }^{3}$

\section{Correspondence:}

Dr. Shaili Pradhan

Periodontology and Oral Implantology Unit, Department of Dental Surgery, National Academy of Medical Sciences, Bir Hospital,

Kathmandu, Nepal.

email: shaili_p@yahoo.com

\section{Citation}

Pradhan S, Shrestha R, Gorkhali RS. Pain Perception after Periodontal Therapies. J Nepal Soc Perio Oral Implantol. 2018;2(2):56-60.
The proper understanding of intensity of pain and variables that affect pain is important as pain may produce emotional responses that could influence hormones, stress level and compliance. ${ }^{4}$ The clinicians can help their patients to build an expectation level and to cope with pain, by providing adequate information about level of pain after dental procedures which will enhance rapport between dentist and patient. $^{5}$

Various types of periodontal surgeries, such as soft tissue grafts and osseous surgeries have been reported to produce more discomfort than routine non-surgical therapy. ${ }^{6}$ Many studies have reported low rates of post-operative infection and complications after periodontal and implant surgeries. ${ }^{7}$ To our knowledge, there is paucity of data in the Nepalese population on pain perception after different types of periodontal surgeries so it is evaluated in this study.

\section{MATERIALS AND METHODS}

The minimum sample size was computed using the formula

$$
\mathrm{n}=\mathrm{z}^{2} \quad \frac{\mathrm{PQ}}{\mathrm{d}^{2}}=63
$$

where:

$\mathrm{n}=$ sample size; $\mathrm{z}=$ standard normal deviate of 1.96 for 
a confidence level set at $95 \% ; \mathrm{P}=$ the prevalence is set at 79.5\%5; $\mathrm{Q}=100-\mathrm{P}=100-79.5=20.5$ and $\mathrm{d}=10$.

A total of 63 systemically healthy subjects: 21 males and 29 females (mean age: 38.84 years ranging from 16-64 years) were recruited from the Periodontology and Oral Implantology Unit, Department of Dental Surgery, National Academy of Medical Sciences, Bir Hospital, Kathmandu. The study was conducted from January 2018 to May 2018 in accordance with Declaration of Helsinki. Demographic information for the patients such as sex and age were taken from patients' records.

All subjects were verbally informed and written informed consent was collected for participation in the study. Subjects with potentially contributory dental conditions (such as active orthodontic treatment, pulpal pathology), pregnancy, immunocompromised subjects, systemically unhealthy patients, patient under analgesic therapy for other diseases and patients taking any additional remedies other than prescribed medication for pain control were excluded from the study.

Phase I therapy (oral hygiene instructions, full-mouth supragingival and subgingival scaling and root planing under local anaesthesia, and occlusal adjustment) was initiated to all the subjects prior to the surgical periodontal therapy. Subjects were scheduled for various periodontal surgeries six to eight weeks following phase I therapy.

All of the surgical procedures were performed following standard protocols under local anaesthesia in strict aseptic condition. Local anaesthetic (LA) used administered was lignocaine $\mathrm{HCl} 2 \%$ with epinephrine 1:2,00,000. Bone graft used was Bio-oss Geistlich Biomaterials, USA) and membrane used was Bio-gide (Geistlich Biomaterials, USA). Periodontal dressing used was Coe-pak (G.C. America Inc., USA) and Barricaid (Dentsply, USA).

Standard post-surgical instructions were given. Subjects who received a free gingival graft were delivered a palatal stent to protect the donor site post-surgery. Antibiotics and analgesics were prescribed, along with chlorhexidine digluconate rinses (0.2\%) 24 hours after surgery twice daily for two weeks. Periodontal dressing and sutures were removed 10 days post-operatively in regenerative surgical cases and seven days post-operatively in other cases. Each subject was reinforced for proper oral hygiene measures.

The data was entered in the questionnaire. The surgeon filled in the data collection form on the type of surgery performed, the duration of surgery, the quantity of anaesthesia used, the number of teeth treated, the arch treated and whether or not periodontal dressings were used. The subjects were instructed to chart their perceptions of pain using a Visual Analog Scale (VAS) with a range of equal units from 0 to 10 , with 0 designated as no pain and 10 for severe excruciating pain. Statistical analysis was done by SPSS 23 software. The mean prevalence of pain between two groups was done by unpaired t test. For all tests, $\mathrm{P}<0.05$ was considered significant. The obtained results were described and plotted.

\section{RESULTS}

A total of 63 surgeries in 50 subjects were included in this study (Table 1). The average age at assessment was 37.84 years, ranging from 16 to 64 years. Males made up $44 \%$ of the sample. Twenty eight subjects were under the age of 40 years.

The surgeries were classified into three types. Their frequency and distribution are listed in Table 2. As listed in Figure 1, the highest mean VAS score was found in open flap debridement (OFD, 2.74), followed by periodontal plastic surgery (2.5) and the lowest mean VAS score was found in resective and regenerative surgery (2.13).

The frequency distribution of various scores is presented in Figure 2. The highest VAS score was 5, whereas the lowest 0. Maximum number of subjects experienced pain of VAS score $2(38.1 \%)$

Among different parameters based on age, sex, periodontal dressing, operator, number of teeth, arch, amount of local anaesthesia and time, statistically different values were found only in sex and the amount of LA used (Table 3). The mean VAS score in subjects less than 40 years of age was

Table 1: Frequency and distribution of different parameters. Parameter (Average) $\mathrm{n}(\%)$

\begin{tabular}{|c|c|}
\hline \multicolumn{2}{|c|}{ Age (37.84 years) } \\
\hline$\leq 40$ years & $28(56.0 \%)$ \\
\hline$>40$ years & $22(44.0 \%)$ \\
\hline \multicolumn{2}{|l|}{ Gender } \\
\hline Male & 22 (44.0\%) \\
\hline Female & $28(56.0 \%)$ \\
\hline \multicolumn{2}{|c|}{ Time (hours) } \\
\hline$\leq 1.5$ & $16(25.4 \%)$ \\
\hline$>1.5$ & 47 (74.6\%) \\
\hline \multicolumn{2}{|c|}{ Periodontal dressing } \\
\hline Yes & $26(41.3 \%)$ \\
\hline No & $37(58.7 \%)$ \\
\hline \multicolumn{2}{|l|}{ Arch } \\
\hline Maxillary & $30(47.6 \%)$ \\
\hline Mandibular & $33(52.4 \%)$ \\
\hline \multicolumn{2}{|l|}{ LA (4.8 ml) } \\
\hline$\leq 3$ & $38(60.3 \%)$ \\
\hline$>3$ & $25(39.7 \%)$ \\
\hline
\end{tabular}


Table 2: VAS score in different types of surgeries.

\begin{tabular}{|l|l|c|c|}
\hline SN & \multicolumn{1}{|c|}{ Type of surgery } & n (\%) & Mean VAS score \\
\hline 1. & Open flap debridement & $31(49.2 \%)$ & 2.74 \\
\hline 2. & Resective and regenerative surgery & $22(34.9)$ & 2.13 \\
\hline 3. & Periodontal plastic surgery & $10(15.9 \%)$ & 2.5 \\
\hline
\end{tabular}

Table 3: Mean VAS Score of the observed data with $\mathrm{p}$ value.

\begin{tabular}{|c|c|c|c|}
\hline Parameter & Type of surgery & Mean VAS Score & $P$ value \\
\hline \multirow{2}{*}{ Age } & $\leq 40$ & 2.41 & \multirow{2}{*}{0.58} \\
\hline & $>40$ & 2.59 & \\
\hline \multirow{2}{*}{ Sex } & Male & 2.14 & \multirow{2}{*}{0.04} \\
\hline & Female & 2.77 & \\
\hline \multirow{2}{*}{ Periodontal dressing } & Given & 2.34 & \multirow{2}{*}{0.43} \\
\hline & Not given & 2.59 & \\
\hline \multirow{2}{*}{ Arch } & Maxillary & 2.34 & \multirow{2}{*}{0.52} \\
\hline & Mandibular & 2.60 & \\
\hline \multirow{2}{*}{ Amount of LA(ml) } & $\leq 3$ & 2.18 & \multirow{2}{*}{0.012} \\
\hline & $>3$ & 2.96 & \\
\hline \multirow{3}{*}{ Time(hours) } & $\leq 2$ & 2.44 & \multirow{3}{*}{0.22} \\
\hline & $>2$ & 3.11 & \\
\hline & $>2$ & 3.11 & \\
\hline
\end{tabular}

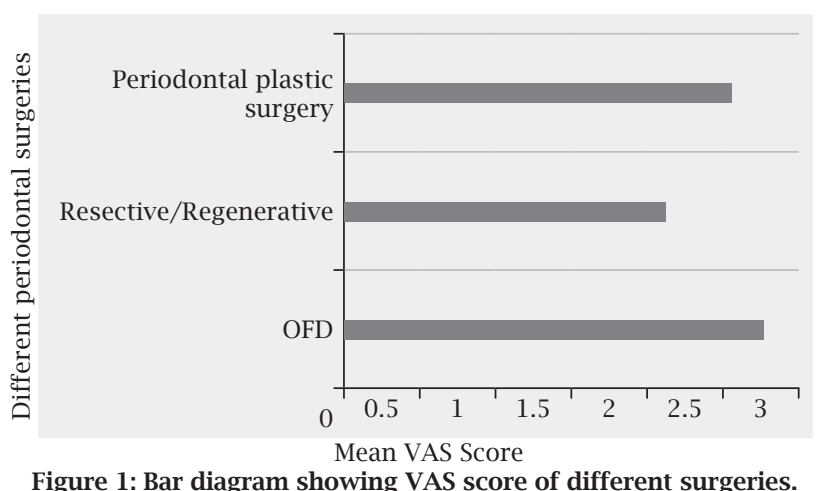

less than those who were above 40. Males showed a mean VAS score of 2.14 and females showed a mean score of 2.77. This showed a statistically significant difference with $P$ value of 0.04 . Mean VAS score was higher when periodontal dressing was placed, when the procedure was carried out in the mandibular arch and when the duration of the surgery was more than two hours. However, the difference failed to show any statistical significance. When larger amount of anaesthesia was used ( $>3 \mathrm{ml}$ ), the mean VAS score obtained was 2.96 , which was statistically significant.

\section{DISCUSSION}

The mean value of VAS for different periodontal surgeries was 2.49. This is in agreement with pain studies on "straight forward" implant installation by Hashem et al (2006), ${ }^{7}$ Al-Khabbaz et al (2007), ${ }^{8}$ on periodontal flap surgical procedures by Fardal and McCulloch $(2012)^{9}$ and on both by Tan et al (2014). ${ }^{10}$ The study done by Al Hamdan $(2009)^{11}$

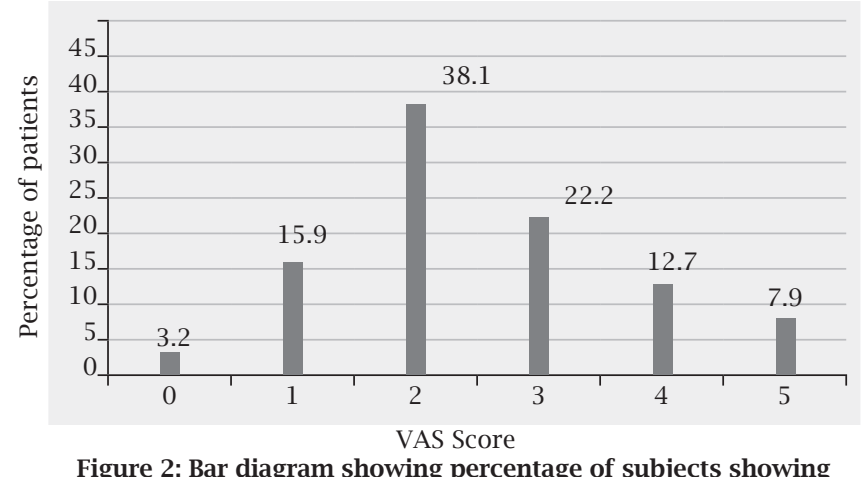

Figure 2: Bar diagram showing percentage of subjects showing different VAS Scores.

also indicated that most of the patients reported mild levels of pain and discomfort following surgical and non-surgical therapy. Mei et al (2016) $)^{5}$ demonstrated that $70 \%$ of the subjects had no or mild pain following periodontal surgeries.

Pain and different surgeries: The study showed highest mean VAS in OFD (2.74), periodontal plastic surgery (2.5) and the lowest in Resective and Regenerative procedures (2.13). A study done by Fardal and McCulloh (2012) ${ }^{9}$ showed that there was no significant differences of VAS scores for perception of discomfort for periodontal surgery $(9.9 \pm 17.0)$ compared to implant surgery $(16.7 \pm 24.2 ; \mathrm{P}>0.2)$. Tan et al $(2014)^{10}$ categorised three surgery types: crown lengthening $(\mathrm{CL})$, OFD and implant and found the difference to be significant after adjustment with other important confounders. They suggested that implant represents a surgical procedure with minor morbidity compared to the periodontal surgical procedures (CL and OFD). In contrast, the study done 
by Al Hamdan (2009)11 found that surgical procedures (including open flap curettage, frenectomy, bone and soft tissue augmentation, crown lengthening, gingivectomy and corticotomy) had the highest pain score, followed by implant surgeries. In another analysis by Canacki and Canacki (2007), ${ }^{13}$ there was no statistically significant differences between the subjects' discomfort levels associated with the four therapy types during periodontal treatment (OFD; Gingivectomy: GV; Modified Widman Flap: MWF and SRP). However, postoperative pain was significantly higher for OFD $(\mathrm{P}<0.01)$ and GV $(\mathrm{P}<0.05)$ procedures than for SRP and MWF procedures. Contradictory to the results found in this study, the study done by Curtis et al $(1985)^{3}$ showed that mucogingival surgery was associated with approximately 3.5 times as much pain as osseous surgery and six times as much pain as soft tissue surgery. Soft tissue surgery was not significantly associated with pain, but osseous and mucogingival surgery were both associated with increased pain.

Pain and Age: The study as shown in Table 3 showed more pain perception in individuals above 40. However, the difference was not statistically significant. Beaudette et al $(2018)^{14}$ demonstrated statistically significant difference in pain perception in older individuals experiencing decreased pain perception. Similar to current findings, Al Hamdan (2009) ${ }^{11}$ study showed subjects between 36-45 years old had more pain than those who were between 18-25 years. Studies by Canakci and Canakci (2007) ${ }^{13}$ and Fardal and Johannessen $(2002)^{15}$ reported that VAS scores decreased with increasing age. Urban and Wenzel $(2010)^{12}$ reported that subjects more than 50 years experienced less pain. Age is one of the biological factors that has been discussed as important for pain experience. ${ }^{15}$ Elderly people are usually more tolerant to pain. The reason for higher pain threshold in elderly subjects may be the consequence of biological phenomenon of tissue changes such as reduced vascularity, fatty degeneration of bone tissue, and secondary dentine formation. ${ }^{15}$

Pain and Sex: The study showed statistically significant differences between male and female, with females showing greater VAS Score. Curtis et al (1984) $)^{3}$ stated that males had significantly less pain than females. In contrast, Canacki and Canacki (2007) ${ }^{13}$ showed no differences in terms of subjects' discomfort during periodontal treatments. Similar findings were seen in studies done by Mei et al (2016), ${ }^{5}$ Lopez et al $(2011)^{16}$ and Dal Pra and Strahan (1972). ${ }^{17}$ Eli et al (2003) ${ }^{4}$ hypothesised that differences in sex and experience of pain is possible due to the reported sex differences in dental fear. However, contrasting findings are possible. Interpretation of VAS score varies with individual. The same score could mean different levels of pain in males and females. ${ }^{14}$

Pain and Periodontal dressing: The present study could not demonstrate any difference in pain with or without the placement of periodontal dressing. Jones and Cassingham $(1979)^{18}$ investigated the effect of periodontal dressings on healing following periodontal surgery. They reported no difference in the parameters of healing between surgically treated quadrants where periodontal dressings were or were not used, but their subjects rather reported an increased discomfort in terms of frequency and severity when a periodontal dressing was placed.

Pain and Location: The study showed statistically insignificant difference in pain in maxilla and mandible. Del Pra and Strahan (1972) ${ }^{17}$ also found no difference in postoperative pain related to the location of surgery.

Pain and LA: This study showed statisitically significant difference in pain perception based on the quantity of anaesthesia used with increased LA volume caused more pain. The result is consistent with the studies done by Sanchez-Siles et al (2014) ${ }^{19}$ and Mei et al $(2016)^{5}$ where the pain and swelling scores in subjects receiving large volume of LA was significantly higher than those receiving small volume of LA. Sanchez-Siles et al (2014) ${ }^{19}$ postulated that the administration of high-volume (>7.2 mL) of anaesthetic agent is associated with increased local tissue distension, including epithelium connective tissue, ligaments and muscles. Injected drug volume is reabsorbed with local vasodilatation acting as a compensatory counterpart. This results in inflammation and the release of proinflammatory mediators, which further entails the stimulation of the nociceptive amielinic nerve endings, causing pain. The benefits of limiting anaesthetic volume include the absence of intraoperative pain, less postoperative pain and swelling, improved subject satisfaction, a lesser use of anti-inflammatory medication and less events of side effects due to overdose of local anesthetics.

Pain and Duration: The study showed surgical procedures with longer duration of time resulted in more pain. However, statistically there was no difference. In a study done by Griffin et al (2006), ${ }^{20}$ the duration of surgical procedures (gingival augmentation) was highly related with pain or swelling post-surgically $(\mathrm{P}=0.001)$. Mei et al $(2016)^{5}$ demonstrated statistically significant difference with moderate to severe pain being experienced on longer duration. Similarly, Tan et al $(2014)^{10}$ also showed results with longer duration of surgery (60 minutes or more) resulting in statistically higher VAS scores for swelling and bruising on the day of surgery itself, when compared to those who had undergone procedures done within 60 minutes. 
The results of this study, although observational in nature, provide an insight in evaluating the level of pain perception and also help in determining the factors that influence pain perception. Considering the low mean VAS score, strong analgesics especially those types containing codeine need not be preferred to prescribe after periodontal surgeries. Periodontal treatment is experienced as painful by substantial number of subjects. The fear of pain resulting from surgery can demotivate the subject to undergo treatment and likely cause the subjects to deny the treatment. Therefore, the dentist should enquire the pain responses during and after treatment and relate the degree of pain according to sex, age and therapy type. Proper understanding of the variables that affect pain is important as they may produce emotional responses that could influence compliance and result of the therapy. Inadvertent use of large dose of anaesthetics assuming high anticipation of pain should be discouraged as the increase in volume relates to increased pain.
The limitations of the study includes small sample size, disproportionate distribution of sample among various groups and lack of assessment of other parameters such as subjects' pre-surgical anxiety scores, smoking, type of incisions, pain subsequent to surgery and consideration of other complication such as bleeding and swelling.

\section{CONCLUSION}

Within the limitations of this study, we can conclude from our investigation that there is low pain perception after different periodontal surgery as measured by VAS. No statistically significant difference in pain was found on the basis of age, sex, duration, location, number of teeth, periodontal dressing and operator. However, there is an obvious relationship between pain and sex and the amount of local anaesthetics used in the procedure.

\section{REFERENCES}

1. The International Association for the Study of Pain (IASP). Pain 1979;6:250.

2. Eli I, Baht R, Kozlovsky A, Simon H. Effect of gender on acute pain prediction and memory in periodontal surgery. Eur Jour Oral Sci. 2000;108:99-103.

3. Curtis JW Jr, McLain JB, Hutchinson RA. The incidence and severity of complications and pain following periodontal surgery. J Periodontol. 1985;56(10):597-601.

4. Eli I, Schwartz-Arad D, Baht R, Ben-Tuvim H. Effect of anxiety on the experience of pain in implant insertion. Clin Oral Implants Res. 2003;14:115-8.

5. Mei CC, Lee FY, Yeh HC. Assessment of pain perception following periodontal and implant surgeries. J Clin Periodontol. 2016;43(12):1151-9.

6. Matthews DC, McCulloch CA. Evaluating patient perceptions as short-term outcomes of periodontal treatment: a comparison of surgical and non-surgical therapy. J Periodontol. 1993;64:990-7.

7. Hashem AA, Claffey NM, O'Connell B. Pain and anxiety following the placement of dental implants. Int J Oral Maxillofac Implant. 2006; 21:943-50.

8. Al-Khabbaz AK, Griffin TJ, Al-Shammari KF. Assessment of pain associated with the surgical placement of dental implants. J Periodontol. 2007;78:239-46.

9. Fardal O, McCulloch CA. Impact of anxiety on pain perception associated with periodontal and implant surgery in a private practice. J Periodontol. 2012;83:1079-85.

10. Tan WC, Krishnaswamy G, Ong MMA, Lang NP. Patient-reported outcome measures after routine periodontal and implant surgical procedures. J Clin Periodontol. 2014;41: 618-24.

11. Al-Hamdan K. Pain perception following different periodontal procedures. Pak Oral Dental J. 2009;29:63-8.

12. Urban T, Wenzel A. Discomfort experienced after immediate implant placement associated with three different regenerative techniques. Clin Oral Implants Res. 2010;21:1271-7.

13. Canakci CF, Varol Canakci V. Pain experienced by patients undergoing different periodontal therapies. J Am Dent Assoc. 2007;138:1563-73.

14. Beaudette JR, Fritz PC, Sullivan PJ, Piccini A, Ward WE. Investigation of factors that influence pain experienced and the use of pain medication following periodontal surgery. J Clin Periodontol. 2018;45:578-85.

15. Fardal Q, Johannessen AC, Linden G. Patient perception of periodontal therapy completed in a periodontal practice. J Periodontal. 2002;73:1060-6.

16. Lopez A, Nart J, Santos A, Alcazar J, Freixa O. Assessment of morbidity after periodontal resective surgery. J Periodontol. $2011 ; 82: 1563-9$.

17. Dal Pra DJ, Strahan JD. A clinical evaluation of the benefits of a course of oral penicillin following periodontal surgery. Aust Dent J. 1972;17:219-21.

18. Jones TM, Cassingham RJ. Comparison of healing following periodontal surgery with and without dressings in humans. J Periodontol. 1979;50:387-93.

19. Sanchez-Siles M, Torres-Diez LC, Camacho-Alonso F, Salazar-Sanchez N, Ballester Ferrandis JF. High volume local anesthesia as a postoperative factor of pain and swelling in dental implants. Clin Implant Dent R. 2014;16:429-34.

20. Griffin TJ, Cheung WS, Zavras AI, Damoulis PD. Postoperative complications following gingival augmentation procedures. J Periodontol. 2006;77:2070-9. 